\title{
Infant recognition of the correspondence between photographs and caricatures of human faces
}

\author{
DONALD J. TYRRELL, JANE T. ANDERSON, MARIANN CLUBB, and ANNE BRADBURY \\ Franklin \& Marshall College, Lancaster, Pennsylvania
}

In two separate experiments, infants were presented with two trials of a novelty preference task. They were familiarized with either a caricature or a photograph of a human face and then, during the novelty test, were presented with a pair of faces, one of which was that of a novel individual. In Experiment 1 the presentation format (caricature or photograph) was constant from familiarization to novelty test, whereas in Experiment 2 the pictures presented during the novelty test were in the format opposite that presented during the familiarization period. The infants in Experiment 1 spent a significant proportion of their looking time fixating the novel face regardless of the presentation format. In Experiment 2, the infants spent significantly more time fixating the novel face only on those trials in which they were familiarized with caricatures and tested with photographs. These results indicate that infants can, under some conditions, recognize a familiar face and discriminate it from a novel face of the same sex even when the presentation format has changed. However, recognition of the correspondence between a caricature and photograph of a specific face is limited to those conditions in which the familiarization stimulus accentuates the distinctive features.

Recent research has demonstrated that within the first year of life infants can recognize varying instances of a specific stimulus such as a human face (e.g., Deloache, Strauss, \& Maynard, 1979; Dirks \& Gibson, 1977). Particularly impressive are the data of Fagan (1976), which led to the conclusion that infants at 4 months of age can identify a specific individual and discriminate that individual from another even though the orientation of the photographs of the faces varied from full front to profile during the interval between familiarization and novelty test. Such a conclusion indicates that infants encode the relevant, invariant features that specify an individual face and recognize those features in the alternate pose.

The purpose of the present experiment was to assess, using the novelty preference format of Fagan (1976), the ability of infants to recognize the correspondence between a caricature and a photograph of an individual face. If, during the familiarization period, the infants encode the relevant, distinctive features that specify a particular individual in one of these presentation formats, they should recognize that individual in the other format, and, if they can discriminate the two faces, they should have a preference for the novel individual.

\footnotetext{
Preparation of this article was supported in part by the Franklin \& Marshall Committee on Grants. Portions of these data were presented at the annual meeting of the Psychonomic Society in San Diego, November, 1983. The authors express their thanks to Susan Marsten for her assistance in the data collection; to Kenneth Ralph and the staff of the Lancaster County Mental Health Association, through which the parents were contacted; and to James Petersen of the Art Department of Franklin \& Marshall College for producing the caricatures. Requests for reprints should be sent to the senior author at Franklin \& Marshall College, Box 3003, Lancaster, PA 17604.
}

An additional feature of the present design was the presentation of two successive trials to each infant. If on the first trial an infant was familiarized with a caricature and tested with photographs, the order was reversed on the second trial. This provided the opportunity to compare performance with both presentation formats and both orders of change (i.e., caricature to photograph and photograph to caricature).

This is of interest because Gibson (1971) and Perkins (1975) argued for the superfidelity of caricatures, due to the exaggeration of the most salient distinctive features combined with the deletion of irrelevant features. If this argument is correct, the infants should have a higher probability of attending to the distinctive features when presented with caricatures, rather than photographs, during familiarization. According to Fagan (1977), an increased probability of attending to the relevant features during familiarization should lead to increased preference for novelty during the novelty test. Thus, the infants should have a greater preference for novelty when switched from caricatures to photographs than in the opposite condition. This asymmetrical preference should not occur if the presentation format is constant throughout the trial, since any feature attended to during familiarization would be available during the novelty test regardless of the stimulus format.

On the other hand, Hagen and Perkins (1983) and Tversky and Baratz (1985) concluded that photographs are processed faster and remembered better than are caricatures. If this is correct, the infants' performance should be improved when they were familiarized with photographs, regardless of the presentation format during the test. This improvement should occur because, with equal time available for encoding during the familiariza- 
tion phase, the increased efficiency of encoding with photographs would lead to increases in preference for novelty (Werner \& Perlmutter, 1979).

\section{EXPERIMENT 1}

The purpose of this experiment was to assess the infants' ability to recognize the familiar face and to discriminate it from the novel face when there was no change in the stimulus format from familiarization to novelty test. Each infant was tested on two successive trials, one with each stimulus type, to allow a comparison of preference for novelty between the two types.

\section{Method}

Subjects. A total of 28 infants were tested. Four were eliminated due to apparatus failure $(n=1)$, uncooperative behavior $(n=2)$, and position preference $(n=1)$. Therefore, the final sample consisted of 24 infants with a mean age of 30 weeks (range, 24 to 36 weeks). These infants were randomly assigned to one of four experimental conditions representing the two orders of presentation format with both male and female stimulus faces. The infants were selected from a pool of parents who responded positively to a request for volunteers sent by the local County Mental Health Association.

Stimuli and Apparatus. The stimuli were presented in an apparatus modeled after that described by Fagan (1970), the essentials of which included a portable testing chamber that contained a rotating "stage" upon which the stimuli were mounted. With the infant seated on a parent's lap and with the stage in the viewing position, the stimuli were approximately $35 \mathrm{~cm}$ from the infant's face. A small $(.75-\mathrm{cm})$ peephole through which the infant's pupils could be observed was located midway between two $17 \times 17 \mathrm{~cm}$ stimulus plaques, which were affixed to the stage $35 \mathrm{~cm}$ apart (center to center). Superimpositions of the stimulus reflection on the pupil of either eye were timed.

From a pool of 20 male and 20 female faces for which both black and white photographs and line-drawn caricatures were available, four instances of each sex were selected. Two pairs of faces were formed within each sex. Fred Astaire and Winston Churchill formed one pair of male faces, and Henry Fonda and Nikita Khrushchev formed the second. The female faces included Marlene Dietrich and Ethel Merman in one pair, and Marilyn Monroe and Elizabeth Taylor in the other. All stimuli were approximately $15 \mathrm{~cm}$ from chin to crown.

These pictures were mounted on white posterboard and affixed to wooden stimulus plaques. An additional wooden plaque, painted the same color as the interior of the apparatus, was attached on the side opposite the single stimulus during the familiarization periods so that the infants would not be distracted by the Velcro strips that secured the plaques.

Procedure. All infants were tested in their homes. After the procedure was explained to the parents, and their permission to proceed was obtained, the infant was seated on the lap of a parent. When the infant was seated comfortably in front of the apparatus, the stage was rotated so that the infant could see the picture, which remained in view until the infant had accumulated a total of $20 \mathrm{sec}$ of fixation time. The stage was then rotated to the experimenter's side of the apparatus and the stimulus was placed on the opposite side and rotated back into the infant's view. The timing of fixations was continued as before until another $20 \mathrm{sec}$ of fixation time had accumulated. Thus, each infant amassed $40 \mathrm{sec}$ of time fixating the picture during this familiarization phase before the novelty test period was begun.

At this point the stage was again rotated out of the infant's view and the pictures for the novelty test were mounted and presented to the infant. The timer was started at the first fixation to either picture and continued for $10 \mathrm{sec}$, during which the total time of all fixations directed to each stimulus was recorded. The stage was again rotated out of the infant's view, the position of the pictures was reversed, and a second test period was initiated. This second period was identical to the first with the exception of the lateral position of the pictures. Thus, the en- tire novelty test consisted of a total of $20 \mathrm{sec}$ during which the infant could fixate the stimuli. This reversal of the position of the pictures within each trial allowed an assessment of any position preference, which was considered to be exhibited when $80 \%$ or more of an infant's fixation time was directed to one side over the two periods. Any infant reaching this criterion on either trial was replaced.

Immediately after the conclusion of the first trial, a second trial was presented. The pair of faces presented on this trial were of the same sex as those presented on the first trial, but the presentation format of the familiarization face was changed. Thus, each infant experienced two successive trials: one in which the familiar and novel pictures were caricatures and one in which they were photographs. Half of the infants were presented with caricatures on Trial 1 and photographs on Trial 2. The remaining infants experienced the opposite order.

All stimulus pairs appeared on the first and second trials, and each member of every pair served as the familiarization picture. The observer was unaware of both the specific stimulus being presented during the familiarization period and the positions of the stimuli during the test periods. The primary dependent variable was the total time the infants fixated each stimulus during the two novelty-test periods of each trial. These times were used to calculate the percentage of total looking time spent fixating the novel stimulus on each trial.

\section{Results}

Preliminary analysis of the preferences for novelty indicated no significant effects due either to trials or to sex of stimulus face; therefore, these factors were ignored in the remainder of the analyses.

The overall mean preference for novelty was $61.00 \%$ $(S D=18.82)$ when the infants were tested with caricatures, and $68.06 \%(S D=12.00)$ when they were tested with photographs. These are both significantly different from chance expectations of $50 \%[t(23)=2.86, p<.01$, and $t(23)=7.36, p<.001$, respectively]. Finally, a $t$ test for related measures indicated that these means were not significantly different from each other $[t(23)=1.62$, $p>.10]$. Thus, there were no differences in performance as a function of the presentation format.

\section{Discussion}

The significant preferences for novelty regardless of the stimulus presentation format indicate that under both formats the infants were able to recognize the familiar face and discriminate it from the novel face. The lack of difference in the performance levels between the two conditions is inconsistent with the expectation based upon the assumption of increased efficiency of encoding with photographs.

\section{EXPERIMENT 2}

Since the infants had significant and equivalent preferences for novelty under both presentation formats, it was possible to employ the same procedure, with the exception of a change in the presentation format from familiarization to novelty test, to measure the ability to recognize the correspondence between the two different formats.

\section{Method}

Subjects. A total of 54 infants were tested. Of these, 14 were eliminated due to apparatus failure $(n=2)$, uncooperative behavior $(n=7)$, and position preferences $(n=5)$. Thus, a total of $\mathbf{4 0}$ infants with a mean age of 33 weeks (range, 30 to 35 weeks) were included in the final sample. 
Procedure. All relevant conditions of Experiment 1 were duplicated in this experiment. However, in all conditions the presentation format was changed between the familiarization and novelty-test phases.

\section{Results}

Preliminary analyses of the preferences for novelty indicated no significant effects associated with either trials or sex of face. Therefore, these factors were ignored in all further analyses.

The mean preference for novelty following familiarization with caricatures and testing with photographs was $60.85 \%(S D=13.31)$, which is significantly greater than the chance expectation of $50 \%[t(39)=5.16, p<.001]$. However, the mean preference following familiarization with photographs and testing with caricatures was $54.74 \%$ $(S D=15.97)$, which was not significantly different from chance $[t(39)=1.88, p>.05]$. A $t$ test for related measures indicated that these means were not significantly different from each other $[t(39)=1.89, p>.05]$.

\section{Discussion}

The percentage of time the infants spent fixating the novel stimulus clearly indicates that the infants significantly preferred the picture of a novel individual if, and only if, they were familiarized with caricatures and tested with photographs. In the opposite condition, the preference for novelty was not different from chance.

\section{GENERAL DISCUSSION}

This asymmetrical preference for the photograph of a novel face after familiarization with a caricature of that face is consistent with the argument of Gibson (1971) and Perkins (1975) that a caricature accentuates the relevant features of a specific face while simultaneously eliminating those features that are not distinctive. Such a condition would increase the probability that the infants would attend to those distinctive features that are relevant to the identification of a particular face. According to Fagan (1977), the increased probability of attending to those relevant features, features which would also exist in the photograph, would lead to an increased preference for the novel stimulus. However, if the photograph is viewed first the infants have a higher probability of attending to those features that are present in the photograph but which are, presumably, irrelevant in specifying a particular individual and which, therefore, might not be present in the caricature. Consequently, because the features they had attended to and encoded would not be present in the caricature, the infants would be unable to recognize the correspondence between the face in the caricature and that in the photograph. Therefore, although the infants might be able to discriminate the two caricatures, they would have no basis for identifying the familiar face and would demonstrate no preference for the novel face.

It should be noted that the present argument assumes that infants can discriminate between two caricatures. The results of Experiment 1 make it clear that the infants made such a discrimination. Thus when the stimulus format was not changed, the infants could recognize and discriminate individual faces regardless of the presentation format. Consequently, it appears that their failure to prefer the novel caricature after having been familiarized with a photograph was due not to an inability to discriminate between two caricatures, but to an inability to recognize the correspondence between the caricature and the photograph of the familiarization face.

It should also be noted that although the current data are not consistent with the arguments of either Hagen and Perkins (1983) or Tversky and Baratz (1985) regarding adults' superior identification of and memory for photographs of faces relative to caricatures, they do not necessarily negate them. The superiority of adult identification and memory for faces may rely upon either encoding or retrieval processes that are not operative in infant recognition memory. However, the efficiency of encoding by infants does not vary as a function of the stimulus type.

It is clear that, at least under some conditions, infants can recognize a specific face and discriminate it from another of the same sex even though the format of presentation has changed from familiarization to test. Presumably this ability depends upon the infant's encoding of the invariant, relevant features of the specific face, which is more likely to occur if those features are accentuated during familiarization. This conclusion extends that of Dirks and Gibson (1977), who demonstrated that infant discrimination depended upon gross differences, such as sex. However, Dirks and Gibson used the habituation/dishabituation paradigm, and the novelty-preference paradigm employed in the present study may be more sensitive to infants' discrimination of subtle differences between stimuli.

Finally, if our interpretation is correct, the use of caricatures, which accentuate unique features and their invariant relationships, combined with the procedures and the mathematical model presented by Fagan (1977), may provide an effective technique for assessing the probability with which infants attend to specific features in the human face.

\section{REFERENCES}

Deloache, J. S., Strauss, M. S., \& Maynard, J. (1979). Picture perception in infancy. Infant Behavior \& Development, 2, 77-89.

Dirks, J., \& GiBson, E. (1977). Infants' perception of similarity between live people and their photographs. Child Development, 48 , 124-130.

FAGAN, J. F. (1970). Memory in the infant. Journal of Experimental Child Psychology, 9, 218-226.

FAGAN, J. F. (1976). Infants' recognition of invariant features of faces. Child Development, 47, 627-638.

FAgAN, J. F. (1977). An attention model of infant recognition. Child Development, 48, 345-359.

Grbson, J. J. (1971). The information available in pictures. Leonardo, 4, 27-35.

Hagan, M. A., \&erkins, D. (1983). A refutation of the hypothesis of the superfidelity of caricatures to photographs. Perception, 12, 55-61.

PerkINS, D. (1975). A definition of caricature and recognition. Studies in the Anthropology of Visual Communication, 2, 1-24.

TVersky, B., \& BARATZ, D. (1985). Memory for faces: Are caricatures better than photographs? Memory \& Cognition, 13, 45-49.

Werner, J. S., \& PerLmutter, M. (1979). Development of visual memory in infants. In H. W. Reese \& L. P. Lipsitt (Eds.), Advances in child development and behavior (Vol. 14). New York: Academic Press.

(Manuscript received for publication August 9, 1986.) 